\title{
Split hierarchical variational inequality problems and related problems
}

\author{
Qamrul Hasan Ansari ${ }^{1,2}$, Nimit Nimana ${ }^{3}$ and Narin Petrot ${ }^{3,4^{*}}$
}

\author{
Correspondence: narinp@nu.ac.th \\ ${ }^{3}$ Department of Mathematics, \\ Faculty of Science, Naresuan \\ University, Phitsanulok, 65000, \\ Thailand \\ ${ }^{4}$ Centre of Excellence in \\ Mathematics, CHE, Si Ayutthaya Rd. \\ Bangkok, 10400, Thailand \\ Full list of author information is \\ available at the end of the article
}

\begin{abstract}
The main objective of this paper is to introduce a split hierarchical variational inequality problem. Several related problems are also considered. We propose an iterative method for finding a solution of our problem. The weak convergence of the sequence generated by the proposed method is studied.

MSC: 47H09; 47H10;47J25; 49J40; 65K10

Keywords: split hierarchical variational inequality problem; split common fixed point problem; split convex minimization problem; strongly nonexpansive operators; cutter operators
\end{abstract}

\section{Introduction}

In 1994, Censor and Elfving [1] introduced the following split feasibility problem (in short, SFP): find a point

$$
x^{*} \in C \text { such that } A x^{*} \in Q \text {, }
$$

where $C$ and $Q$ are nonempty closed convex subsets of $\mathbb{R}^{n}$ and $\mathbb{R}^{m}$, respectively, and $A$ is an $m \times n$ matrix. They proposed an algorithm to find the solution of SFP. Their algorithm did not become popular, since it concerns the complicated matrix inverse computations and, subsequently, is considered in the case when $n=m$. Based on these observations, Byrne [2] applied the forward-backward method, a type of projected gradient method, presenting the so-called CQ-iterative procedure, which is defined by

$$
x_{k+1}=P_{C}\left(x_{k}+\gamma A^{\top}\left(P_{Q}-I\right) A x_{k}\right), \quad \forall k \geq 1,
$$

where an initial $x_{1} \in \mathbb{R}^{n}, \gamma \in\left(0,2 /\|A\|^{2}\right)$, and $P_{C}$ and $P_{Q}$ denote the metric projections onto $C$ and $Q$, respectively. The convergence result of the sequence $\left\{x_{k}\right\}_{k=1}^{\infty}$ to a solution of the considered split feasibility problem was presented. Further, Byrne also proposed an application to dynamic emission tomographic image reconstruction. A few years later, Censor et al. $[3,4]$ proposed an incredible application of the split feasibility problem to the inverse problem of intensity-modulated radiation therapy treatment planning. Recently, $\mathrm{Xu}$ [5] considered SFP in the setting of infinite-dimensional Hilbert spaces and established the following CQ-algorithm: Let $H_{1}$ and $H_{2}$ be real Hilbert spaces and $A: H_{1} \rightarrow H_{2}$ be a

\section{Springer}

(c) 2014 Ansari et al.; licensee Springer. This is an Open Access article distributed under the terms of the Creative Commons Attribution License (http://creativecommons.org/licenses/by/2.0), which permits unrestricted use, distribution, and reproduction in any medium, provided the original work is properly cited. 
bounded linear operator. For a given $x_{1} \in H_{1}$, generated a sequence $\left\{x_{k}\right\}_{k=1}^{\infty}$ by the following iterative scheme:

$$
x_{k+1}=P_{C}\left(x_{k}+\gamma A^{*}\left(P_{Q}-I\right) A x_{k}\right), \quad \forall k \geq 1,
$$

where $\gamma \in\left(0,2 /\|A\|^{2}\right)$, and $A^{*}$ is the adjoint operator of $A$. He also proved the weak convergence of the sequence produced by the above procedure to a solution of the SFP. The details of the CQ-algorithm for SFP problem are given in [6]. A comprehensive literature, survey, and references on SFP can be found in [7].

In 2009, Censor and Segal [8] presented an important form of the split feasibility problem called the split common fixed point problem, which is to find a point

$$
x^{*} \in \operatorname{Fix}(T) \text { such that } A x^{*} \in \operatorname{Fix}(S) \text {, }
$$

where $T$ and $S$ are some nonlinear operators on $\mathbb{R}^{n}$ and $\mathbb{R}^{m}$, respectively, and $A$ is a real $m \times n$ matrix. Based on the properties of operators $T$ and $S$, called cutter or directed operators, they presented the following algorithm for solving the split common fixed point problem:

$$
x_{k+1}=T\left(x_{k}+\gamma A^{\top}(S-I) A x_{k}\right), \quad \forall k \geq 1,
$$

where an initial $x_{1} \in \mathbb{R}^{n}, \gamma \in\left(0,2 /\|A\|^{2}\right)$. They also presented a convergence result for this algorithm. Moudafi [9] studied the split common fixed point problem in the context of the demicontractive operators $T$ and $S$ in the setting of infinite-dimensional Hilbert spaces. He established the weak convergence of the sequence generated by his scheme to a solution of the split common fixed point problem.

On the other hand, the theory of variational inequalities is well known and well developed because of its applications in different areas of science, social science, engineering, and management. There are several monographs on variational inequalities, but we mention here a few [10-12]. Let $C$ be a nonempty closed convex subset of a real Hilbert space $H$ and $f: H \rightarrow H$ be an operator. The variational inequality problem defined by $C$ and $f$ is to find $x^{*} \in C$ such that

$(\operatorname{VIP}(C ; f)) \quad\left\langle f\left(x^{*}\right), x-x^{*}\right\rangle \geq 0, \quad$ for all $x \in C$.

Another problem closely related to $\operatorname{VIP}(C ; f)$ is known as the Minty variational inequality problem: find $x^{*} \in C$ such that

$(\operatorname{MVIP}(C ; f)) \quad\left\langle f(x), x-x^{*}\right) \geq 0, \quad$ for all $x \in C$.

The trivial unlikeness of two proposed problems is the linearity of variational inequalities. In fact, the Minty variational inequality $\operatorname{MVIP}(C ; f)$ is linear but the variational inequality $\operatorname{VIP}(C ; f)$ is not. However, under the (hemi)continuity and monotonicity of $f$, the solution sets of these problems are the same (see [13, Lemma 1]).

If the constrained set $C$ in variational inequality formulations is a set of fixed points of an operator, then the variational inequality problem is known as hierarchical variational inequality problem. 
Let $T: H \rightarrow H$ be a nonlinear operator with the set of fixed points $\operatorname{Fix}(T) \neq \emptyset$ and $f$ : $H \rightarrow H$ be an operator. The hierarchical variational inequality problem is to find $x^{*} \in$ Fix $(T)$ such that

$$
\left\langle f\left(x^{*}\right), x-x^{*}\right\rangle \geq 0, \quad \text { for all } x \in \operatorname{Fix}(T) .
$$

A closely related problem to hierarchical variational inequality problem is the hierarchical Minty variational inequality problem: find $x^{*} \in \operatorname{Fix}(T)$ such that

$$
\left\langle f(x), x-x^{*}\right\rangle \geq 0, \quad \text { for all } x \in \operatorname{Fix}(T) .
$$

In the recent past, several methods for solving hierarchical variational inequalities have been investigated in the literature; see, for example, [14-19] and the references therein.

The goal of this paper is to introduce a split-type problem, by combining a split fixed point problem and a hierarchical variational inequality problem. The considered problem can be applied to solve many existing problems. We present an iterative procedure for finding a solution of the proposed problem and show that under some suitable assumptions, the sequence generated by our algorithm converges weakly to a solution of the considered problem.

The rest of this paper is divided into four sections. In Section 2, we recall and state preliminaries on numerous nonlinear operators and their useful properties. Section 3 we divide in two subsections, that is, first, we present a split problem, called the split hierarchical Minty variational inequality problem. We subsequently propose an algorithm for solving our problem and establish a convergence result under some assumptions. Second, we present the split hierarchical variational inequality problem. In the last section, we investigate some related problems, where we can apply the considered problem.

\section{Preliminaries}

Let $H$ be a real Hilbert space whose inner product and norm are denoted by $\langle\cdot, \cdot\rangle$ and $\|\cdot\|$, respectively. The strong convergence and weak convergence of a sequence $\left\{x_{k}\right\}_{k=1}^{\infty}$ to $x \in H$ are denoted by $x_{k} \rightarrow x$ and $x_{k} \rightarrow x$, respectively. Let $T: H \rightarrow H$ be an operator. We denote by $R(T)$ the range of $T$, and by $\operatorname{Fix}(T)$ the set of all fixed points of $T$, that is, $\operatorname{Fix}(T)=\{x \in H: x=T x\}$. The operator $T$ is said to be nonexpansive if for all $x, y \in H$, $\|T x-T y\| \leq\|x-y\| ;$ strongly nonexpansive $[20,21]$ if $T$ is nonexpansive and for all bounded sequences $\left\{x_{k}\right\}_{k=1}^{\infty},\left\{y_{k}\right\}_{k=1}^{\infty}$ in $H$, the condition $\lim _{k \rightarrow \infty}\left(\left\|x_{k}-y_{k}\right\|-\left\|T x_{k}-T y_{k}\right\|\right)=0$ implies $\lim _{k \rightarrow \infty}\left\|\left(x_{k}-y_{k}\right)-\left(T x_{k}-T y_{k}\right)\right\|=0$; averaged nonexpansive if for all $x, y \in H, T=(1-$ $\alpha) I+\alpha S$ holds for a nonexpansive operator $S: H \rightarrow H$ and $\alpha \in(0,1)$; firmly nonexpansive if $2 T-I$ is nonexpansive, or equivalently for all $x, y \in H,\|T x-T y\|^{2} \leq\langle x-y, T x-T y\rangle$; cutter [21] if $\langle x-T x, z-T x\rangle \leq 0$ for all $x \in H$ and all $z \in \operatorname{Fix}(T)$; monotone if $\langle T x-T y, x-y\rangle \geq 0$, for all $x, y \in H$; $\alpha$-inverse strongly monotone (or $\alpha$-cocoercive) if there exists a positive real number $\alpha$ such that $\langle T x-T y, x-y\rangle \geq \alpha\|T x-T y\|^{2}$, for all $x, y \in H$.

Let $B: H \rightarrow 2^{H}$ be a set-valued operator, we define a graph of $B$ by $\{(x, y) \in H \times H$ : $y \in B(x)\}$ and an inverse operator of $B$, denoted $B^{-1}$, by $\{(y, x) \in H \times H: x \in B(y)\}$. A setvalued operator $B: H \rightarrow 2^{H}$ is called monotone if for all $x, y \in H, u \in B(x)$ and $v \in B(y)$ such that $\langle x-y, u-v\rangle \geq 0$. A monotone operator $B$ is said to be maximal monotone if there exists no other monotone operator such that its graph properly contains the graph 
of $B$. For a maximal monotone operator $B$, we know that for each $x \in H$ and a positive real number $\sigma$, there is a unique $z \in H$ such that $x \in(I+\sigma B) z$. We define the resolvent of $B$ with parameter $\sigma$ by $J_{\sigma}^{B}:=(I+\sigma B)^{-1}$. It is well known that the resolvent is a single-valued and firmly nonexpansive operator.

Remark 2.1 It can be seen that the class of averaged nonexpansive operators is a proper subclass of the class of strongly nonexpansive operators. Since any firmly nonexpansive operator is an averaged nonexpansive operator, it is clear that a class of firmly nonexpansive operators is contained in the class of strongly nonexpansive operators. Further, a firmly nonexpansive operator with fixed point is cutter. However, a nonexpansive cutter operator need not to be firmly nonexpansive; for further details, see [21].

The following well-known lemma is due to Opial [22].

Lemma 2.2 (Demiclosedness principle) [22, Lemma 2] Let C be a nonempty closed convex subset of a real Hilbert space $H$ and $T: C \rightarrow H$ be a nonexpansive operator. If the sequence $\left\{x_{k}\right\}_{k=1}^{\infty} \subseteq C$ converges weakly to an element $x \in C$ and the sequence $\left\{x_{k}-T x_{k}\right\}_{k=1}^{\infty}$ converges strongly to 0, then $x$ is a fixed point of the operator $T$.

To prove the main theorem of this paper, we need the following lemma.

Lemma 2.3 [23, Section 2.2.1, Lemma 2] Assume that $\left\{a_{k}\right\}_{k=1}^{\infty}$ and $\left\{b_{k}\right\}_{k=1}^{\infty}$ are nonnegative real sequences such that $a_{k+1} \leq a_{k}+b_{k}$. If $\sum_{k=1}^{\infty} b_{k}<\infty$, then $\lim _{k \rightarrow \infty} a_{k}$ exists.

The following lemma can be immediately obtained by the properties of an inner product.

Lemma 2.4 Let $H$ be a real Hilbert space $H$. Then, for all $x, y \in H$,

$$
\|x-y\|^{2} \leq\|x\|^{2}+2\langle y, y-x\rangle \text {. }
$$

\section{Split hierarchical variational inequality problems and convergent results}

In this section we introduce split hierarchical variational inequality problems and discuss some related problems. Further, we propose an iterative method for finding a solution of the hierarchical variational inequality problem and prove the convergence result for the sequence generated by the proposed iterative method.

\subsection{Split hierarchical Minty variational inequality problem}

Let $H_{1}$ and $H_{2}$ be two real Hilbert spaces, $f, T: H_{1} \rightarrow H_{1}$ be operators such that $\operatorname{Fix}(T) \neq \emptyset$, and $h, S: H_{2} \rightarrow H_{2}$ be operators such that $\operatorname{Fix}(S) \neq \emptyset$. Let $A: H_{1} \rightarrow H_{2}$ be an operator with $R(A) \cap \operatorname{Fix}(S) \neq \emptyset$. The split hierarchical Minty variational inequality problem (in short, SHMVIP) is to find $x^{*} \in \operatorname{Fix}(T)$ such that

$$
\left\langle f(x), x-x^{*}\right\rangle \geq 0, \quad \text { for all } x \in \operatorname{Fix}(T)
$$

and such that $A x^{*} \in \operatorname{Fix}(S)$ satisfies

$$
\left\langle h(y), y-A x^{*}\right\rangle \geq 0, \quad \text { for all } y \in \operatorname{Fix}(S) .
$$


The solution set of SHMVIP (3.1)-(3.2) is denoted by $\Gamma$, that is,

$$
\Gamma:=\{x \text { which solves }(3.1): A x \text { solves }(3.2)\}
$$

We note that the problems (3.1) and (3.2) are nothing but hierarchical Minty variational inequality problems. If $f$ and $h$ are zero operators, that is, $f \equiv h \equiv 0$, then SHMVIP (3.1) and (3.2) reduces to the split fixed point problem (1.2). Moreover, SHMVIP (3.1) and (3.2) can be applied to several existing split-type problems, which we will discuss in Section 4 . Inspired by the iterative scheme (1.3) for solving split common fixed point problem (1.2) and the existing algorithms, a generalization of the projected gradient method, for solving the hierarchical variational inequality problem of Yamada [19] and Iiduka [16], we now present an iterative algorithm for solving HMVIP (3.1)-(3.2).

\section{Algorithm 3.1}

Initialization: Choose $\left\{\alpha_{k}\right\}_{k=1}^{\infty},\left\{\beta_{k}\right\}_{k=1}^{\infty} \subset(0,+\infty)$. Take arbitrary $x_{1} \in H_{1}$. Iterative Step: For a given current iterate $x_{k} \in H_{1}$, compute

$$
y_{k}:=x_{k}-\gamma A^{*}\left(I-S\left(I-\beta_{k} h\right)\right) A x_{k} \text {, }
$$

where $\gamma \in\left(0, \frac{2}{\|A\|^{2}}\right)$ and define $x_{k+1} \in H_{1}$ by

$$
x_{k+1}:=T\left(I-\alpha_{k} f\right) y_{k} .
$$

Update $k:=k+1$

Rest of the section, unless otherwise specified, we assume that $T$ is a strongly nonexpansive operator on $H_{1}$ with $\operatorname{Fix}(T) \neq \emptyset$ and $S$ is a strongly nonexpansive cutter operator on $H_{2}$ with $\operatorname{Fix}(S) \neq \emptyset, f$ (respectively, $h$ ) is a monotone and continuous operator on $H_{1}$ (respectively, $H_{2}$ ) and $A: H_{1} \rightarrow H_{2}$ is a bounded linear operator with $R(A) \cap \operatorname{Fix}(S) \neq \emptyset$.

Remark 3.2 (i) The Algorithm 3.1 can be applied to the iterative scheme (1.3) by setting the operators $f \equiv h \equiv 0$.

(ii) The iterative Algorithm 3.1 extends and develops the algorithms in [24, Algorithm 6.1] and [25, Algorithm (8)] in many aspects. Indeed, the metric projections $P_{C}$ and $P_{Q}$ in [24, Algorithm 6.1] and the resolvent operators $J_{\lambda}^{B_{1}}$ and $J_{\lambda}^{B_{2}}$ in [25, Algorithm (8)] are firmly nonexpansive operators which are special cases of the assumption on the operators $T$ and $S$. Second, its involves control sequences $\left\{\alpha_{k}\right\}_{k=1}^{\infty}$ and $\left\{\beta_{k}\right\}_{k=1}^{\infty}$, while in [24, Algorithm 6.1] and [25, Algorithm (8)] they are constant. Furthermore, we assume $\gamma \in\left(0, \frac{2}{\|A\|^{2}}\right)$, while in [24, Algorithm 6.1] and [25, Algorithm (8)], it was assumed to be in $\left(0, \frac{1}{\|A\|^{2}}\right)$, which clearly is a more restrictive assumption.

(iii) By setting an operator $A$ to be zero operator, the iterative Algorithm 3.1 relates to existing iterative schemes for solving the hierarchical variational inequality problem in, for example, [15, Algorithm 3.1] and [17, Algorithm 5].

The following theorem provides the weak convergence of the sequence generated by the Algorithm 3.1 to an element of $\Gamma$. 
Theorem 3.3 Let a sequence $\left\{x_{k}\right\}_{k=1}^{\infty}$ be generated by Algorithm 3.1 with $x_{1} \in H_{1}$, and let $\left\{\alpha_{k}\right\}_{k=1}^{\infty}$ and $\left\{\beta_{k}\right\}_{k=1}^{\infty} \subset(0,1)$ be sequences such that $\sum_{k=1}^{\infty} \alpha_{k}<\infty$ and $\lim _{k \rightarrow \infty} \beta_{k}=0$. If $\Gamma \neq \emptyset$, then the following statements hold.

(i) If there exists a natural number $k_{0}$ such that

$$
\Gamma \subset \bigcap_{k=k_{0}}^{\infty}\left\{z \in H_{1}:\left\langle h\left(A x_{k}\right), S\left(I-\beta_{k} h\right) A x_{k}-A z\right\rangle \geq 0\right\}
$$

then, for all $k \geq k_{0}$ and $z \in \Gamma$, we have

$$
\left\|y_{k}-z\right\|^{2} \leq\left\|x_{k}-z\right\|^{2}-\gamma\left(2-\gamma\|A\|^{2}\right)\left\|\left(I-S\left(I-\beta_{k} h\right)\right) A x_{k}\right\|^{2} .
$$

(ii) If the sequence $\left\{f\left(y_{k}\right)\right\}_{k=1}^{\infty}$ is bounded, then $\lim _{k \rightarrow \infty}\left\|x_{k}-z\right\|$ exists for all $z \in \Gamma$.

(iii) If the sequence $\left\{h\left(A x_{k}\right)\right\}_{k=1}^{\infty}$ is bounded, then $\lim _{k \rightarrow \infty}\left\|x_{k+1}-x_{k}\right\|=0$,

$\lim _{k \rightarrow \infty}\left\|x_{k}-T x_{k}\right\|=0$, and $\lim _{k \rightarrow \infty}\left\|A x_{k}-S A x_{k}\right\|=0$.

(iv) If $\left\|x_{k+1}-x_{k}\right\|=o\left(\alpha_{k}\right)$, and $\alpha_{k}=o\left(\beta_{k}^{2}\right)$, then the sequence $\left\{x_{k}\right\}_{k=1}^{\infty}$ converges weakly to an element of $\Gamma$.

Proof (i) Let $z \in \Gamma$ be given. Then

$$
\begin{aligned}
\left\|y_{k}-z\right\|^{2}= & \left\|x_{k}-\gamma A^{*}\left(I-S\left(I-\beta_{k} h\right)\right) A x_{k}-z\right\|^{2} \\
= & \left\|x_{k}-z\right\|^{2}+\gamma^{2}\left\|A^{*}\left(I-S\left(I-\beta_{k} h\right)\right) A x_{k}\right\|^{2} \\
& -2 \gamma\left\langle x_{k}-z, A^{*}\left(I-S\left(I-\beta_{k} h\right)\right) A x_{k}\right\rangle \\
\leq & \left\|x_{k}-z\right\|^{2}+\gamma^{2}\|A\|^{2}\left\|\left(I-S\left(I-\beta_{k} h\right)\right) A x_{k}\right\|^{2} \\
& -2 \gamma\left(x_{k}-z, A^{*}\left(I-S\left(I-\beta_{k} h\right)\right) A x_{k}\right\rangle,
\end{aligned}
$$

for all $k \geq 1$. On the other hand, since $S$ is a cutter operator, we have with

$$
\begin{aligned}
\left\langle x_{k}-\right. & \left.z, A^{*}\left(S\left(I-\beta_{k} h\right)-I\right) A x_{k}\right\rangle \\
= & \left\langle A x_{k}-A z,\left(S\left(I-\beta_{k} h\right)-I\right) A x_{k}\right\rangle \\
= & \left\langle S\left(I-\beta_{k} h\right)\left(A x_{k}\right)-A z, S\left(I-\beta_{k} h\right)\left(A x_{k}\right)-A x_{k}\right\rangle \\
& -\left\|\left(S\left(I-\beta_{k} h\right)-I\right) A x_{k}\right\|^{2} \\
= & \left\langle S\left(I-\beta_{k} h\right)\left(A x_{k}\right)-A z, S\left(I-\beta_{k} h\right)\left(A x_{k}\right)-\left(I-\beta_{k} h\right) A x_{k}\right\rangle \\
& -\beta_{k}\left\langle S\left(I-\beta_{k} h\right)\left(A x_{k}\right)-A z, h\left(A x_{k}\right)\right\rangle-\left\|\left(S\left(I-\beta_{k} h\right)-I\right) A x_{k}\right\|^{2} \\
\leq & -\left\|\left(S\left(I-\beta_{k} h\right)-I\right) A x_{k}\right\|^{2},
\end{aligned}
$$

for all $k \geq k_{0}$. By using (3.4), the inequality (3.3) becomes

$$
\begin{aligned}
\left\|y_{k}-z\right\|^{2} \leq & \left\|x_{k}-z\right\|^{2}+\gamma^{2}\|A\|^{2}\left\|\left(S\left(I-\beta_{k} h\right)-I\right) A x_{k}\right\|^{2} \\
& -2 \gamma\left\|\left(S\left(I-\beta_{k} h\right)-I\right) A x_{k}\right\|^{2} \\
= & \left\|x_{k}-z\right\|^{2}-\gamma\left(2-\gamma\|A\|^{2}\right)\left\|\left(S\left(I-\beta_{k} h\right)-I\right) A x_{k}\right\|^{2},
\end{aligned}
$$


for all $k \geq k_{0}$, as required. Furthermore, since $\gamma<\frac{2}{\|A\|^{2}}$, we observe that $\gamma\left(2-\gamma\|A\|^{2}\right)>0$, and hence

$$
\left\|y_{k}-z\right\| \leq\left\|x_{k}-z\right\|, \quad \forall k \geq k_{0} .
$$

(ii) Let $M_{1}:=\sup \left\{\left\|f\left(y_{k}\right)\right\|: k \geq 1\right\}$. For $k \geq k_{0}$, we have $\left\|x_{k+1}-z\right\| \leq\left\|x_{k}-z\right\|+\alpha_{k} M_{1}$. Since $\sum_{k=1}^{\infty} \alpha_{k}<\infty$, by using Lemma 2.3 , we obtain the result that $\lim _{k \rightarrow \infty}\left\|x_{k}-z\right\|$ exists.

(iii) We first show that $\lim _{k \rightarrow \infty}\left\|x_{k}-x_{k+1}\right\|=0$.

Let $w_{k}:=y_{k}-\alpha_{k} f\left(y_{k}\right)$ for all $k \geq 1$. Note that $\left\{w_{k}\right\}_{k=1}^{\infty}$ is a bounded sequence, since for all $k \geq k_{0}$ we know that $\left\|w_{k}-z\right\| \leq\left\|y_{k}-z\right\|+\alpha_{k}\left\|f\left(y_{k}\right)\right\|$ and $\left\{y_{k}\right\}_{k=1}^{\infty}$ is a bounded sequence. By using the nonexpansiveness of $T$ and (3.5), we have

$$
0 \leq\left\|w_{k}-z\right\|-\left\|T w_{k}-T z\right\| \leq\left\|x_{k}-z\right\|+\alpha_{k}\left\|f\left(y_{k}\right)\right\|-\left\|x_{k+1}-z\right\|, \quad \forall k \geq k_{0} .
$$

Subsequently, by the existence of $\lim _{k \rightarrow \infty}\left\|x_{k}-z\right\|$ and the fact that $\alpha_{k} \rightarrow 0$, it follows that

$$
\lim _{k \rightarrow \infty}\left(\left\|w_{k}-z\right\|-\left\|T w_{k}-T z\right\|\right)=0
$$

By the strong nonexpansiveness of $T$ and the boundedness of $\left\{w_{k}\right\}_{k=1}^{\infty}$, we have

$$
\lim _{k \rightarrow \infty}\left\|w_{k}-T w_{k}\right\|=0
$$

On the other hand, by (i), we observe that

$$
\begin{aligned}
\left\|x_{k+1}-z\right\|^{2} \leq & \left\|y_{k}-\alpha_{k} f\left(y_{k}\right)-z\right\|^{2} \\
\leq & \left\|y_{k}-z\right\|^{2}+\alpha_{k}^{2}\left\|f\left(y_{k}\right)\right\|^{2}+2 \alpha_{k}\left\|f\left(y_{k}\right)\right\|\left\|y_{k}-z\right\| \\
\leq & \left\|x_{k}-z\right\|^{2}-\gamma\left(2-\gamma\|A\|^{2}\right)\left\|\left(I-S\left(I-\beta_{k} h\right)\right) A x_{k}\right\|^{2} \\
& +\alpha_{k}\left\|f\left(y_{k}\right)\right\|^{2}+2 \alpha_{k}\left\|f\left(y_{k}\right)\right\|\left\|y_{k}-z\right\|,
\end{aligned}
$$

which is equivalent to

$$
\begin{gathered}
\gamma\left(2-\gamma\|A\|^{2}\right)\left\|\left(I-S\left(I-\beta_{k} h\right)\right) A x_{k}\right\|^{2} \leq \\
\quad\left\|x_{k}-z\right\|^{2}-\left\|x_{k+1}-z\right\|^{2}+\alpha_{k}\left\|f\left(y_{k}\right)\right\|^{2} \\
+2 \alpha_{k}\left\|f\left(y_{k}\right)\right\|\left\|y_{k}-z\right\|,
\end{gathered}
$$

for all $k \geq k_{0}$. Taking the limit as $k \rightarrow \infty$, we get

$$
\lim _{k \rightarrow \infty}\left\|\left(I-S\left(I-\beta_{k} h\right)\right) A x_{k}\right\|=0,
$$

which implies, by the definition of $y_{k}$, that

$$
\lim _{k \rightarrow \infty}\left\|y_{k}-x_{k}\right\|=0
$$

and also

$$
\lim _{k \rightarrow \infty}\left\|w_{k}-x_{k}\right\|=0
$$


These together imply that

$$
\lim _{k \rightarrow \infty}\left\|x_{k}-x_{k+1}\right\| \leq \lim _{k \rightarrow \infty}\left(\left\|x_{k}-w_{k}\right\|+\left\|w_{k}-T w_{k}\right\|\right)=0
$$

as desired.

We now show that $\lim _{k \rightarrow \infty}\left\|x_{k}-T x_{k}\right\|=0$.

Observe that $\left\|x_{k+1}-T y_{k}\right\| \leq \alpha_{k}\left\|f\left(y_{k}\right)\right\| \rightarrow 0$ as $k \rightarrow \infty$. Using this one together with (3.8), (3.9) and the nonexpansiveness of $T$, we obtain

$$
\lim _{k \rightarrow \infty}\left\|x_{k}-T x_{k}\right\|=0
$$

Next, we show that $\lim _{k \rightarrow \infty}\left\|A x_{k}-S A x_{k}\right\|=0$.

Set $z_{k}:=A x_{k}-\beta_{k} h\left(A x_{k}\right)$ for all $k \geq 1$. For every $k \geq k_{0}$, we observe that

$$
0 \leq\left\|z_{k}-A z\right\|-\left\|S z_{k}-S A z\right\| \leq \beta_{k}\left\|h\left(A x_{k}\right)\right\|+\left\|A x_{k}-S z_{k}\right\| .
$$

Thus, the boundedness of $\left\{h\left(A x_{k}\right)\right\}_{k=1}^{\infty}$ and (3.7) yield

$$
\lim _{k \rightarrow \infty}\left(\left\|z_{k}-A z\right\|-\left\|S z_{k}-S A z\right\|\right)=0,
$$

and hence, by the fact that the sequence $\left\{z_{k}\right\}_{k=1}^{\infty}$ is bounded and $S$ is a strongly nonexpansive mapping, we have

$$
\lim _{k \rightarrow \infty}\left\|z_{k}-S z_{k}\right\|=0
$$

We note that $\left\|A x_{k}-z_{k}\right\|=\beta_{k}\left\|h\left(A x_{k}\right)\right\| \rightarrow 0$ as $k \rightarrow \infty$. Also, we observe that

$$
\left\|z_{k}-S A x_{k}\right\| \leq\left\|z_{k}-S z_{k}\right\|+\left\|S\left(A x_{k}-\beta_{k} h\left(A x_{k}\right)\right)-S A x_{k}\right\| \leq\left\|z_{k}-S z_{k}\right\|+\beta_{k}\left\|h\left(A x_{k}\right)\right\|,
$$

for all $k \geq k_{0}$. By using (3.11) and taking the limit as $k \rightarrow \infty$, we get

$$
\lim _{k \rightarrow \infty}\left\|z_{k}-S A x_{k}\right\|=0
$$

Hence, we get

$$
\lim _{k \rightarrow \infty}\left\|A x_{k}-S A x_{k}\right\|=0
$$

as required.

(iv) Since $\left\{x_{k}\right\}_{k=1}^{\infty}$ is a bounded sequence, there exist a subsequence $\left\{x_{k_{j}}\right\}_{j=1}^{\infty}$ of $\left\{x_{k}\right\}_{k=1}^{\infty}$ and $q \in H_{1}$ such that $x_{k_{j}} \rightarrow q \in H_{1}$. By (iii) and the demiclosed principle of the nonexpansive operator $T$, we obtain $q \in \operatorname{Fix}(T)$. We claim that $q \in \operatorname{Fix}(T)$ solves (3.1).

In fact, for $k \geq k_{0}$, we have

$$
\begin{aligned}
\left\|x_{k+1}-z\right\|^{2} & \leq\left\|y_{k}-z\right\|^{2}+2 \alpha_{k}\left\langle f\left(y_{k}\right), z-w_{k}\right\rangle \\
& =\left\|x_{k}-z\right\|^{2}+2 \alpha_{k}\left\langle f\left(y_{k}\right), z-y_{k}\right\rangle+2 \alpha_{k}^{2}\left\|f\left(y_{k}\right)\right\|^{2}
\end{aligned}
$$




$$
\begin{aligned}
= & \left\|x_{k}-z\right\|^{2}+2 \alpha_{k}\left\langle f(z), z-y_{k}\right\rangle \\
& -2 \alpha_{k}\left|f\left(y_{k}\right)-f(z), y_{k}-z\right\rangle+2 \alpha_{k}^{2}\left\|f\left(y_{k}\right)\right\|^{2} \\
\leq & \left\|x_{k}-z\right\|^{2}+2 \alpha_{k}\left\langle f(z), z-y_{k}\right\rangle+2 \alpha_{k}^{2}\left\|f\left(y_{k}\right)\right\|^{2},
\end{aligned}
$$

and then

$$
\begin{aligned}
2\left(f(z), y_{k}-z\right) & \leq \frac{1}{\alpha_{k}}\left(\left\|x_{k}-z\right\|^{2}-\left\|x_{k+1}-z\right\|^{2}\right)+2 \alpha_{k}\left\|f\left(y_{k}\right)\right\|^{2} \\
& \leq \frac{1}{\alpha_{k}}\left(\left\|x_{k}-z\right\|+\left\|x_{k+1}-z\right\|\right)\left(\left\|x_{k}-z\right\|-\left\|x_{k+1}-z\right\|\right)+2 \alpha_{k}\left\|f\left(y_{k}\right)\right\|^{2} \\
& \leq \frac{\left\|x_{k}-x_{k+1}\right\|}{\alpha_{k}} M+2 \alpha_{k}\left\|f\left(y_{k}\right)\right\|^{2},
\end{aligned}
$$

where $M:=\sup \left\{\left\|x_{k}-z\right\|+\left\|x_{k+1}-z\right\|: k \geq 1\right\}<\infty$. Since $x_{k_{j}} \rightarrow q, \alpha_{k} \rightarrow 0$, and $\left\|x_{k+1}-x_{k}\right\|=$ $o\left(\alpha_{k}\right)$, by (3.8), we have $\langle f(z), q-z\rangle \leq 0$ for all $z \in \operatorname{Fix}(T)$ which means that $q \in \operatorname{Fix}(T)$ solves (3.1).

Next, by $A x_{k_{j}} \rightarrow A q \in H_{2}$ together with (iii) and the demiclosedness of the nonexpansive operator $S$, we know that $A q \in \operatorname{Fix}(S)$. We show that such $A q \in \operatorname{Fix}(S)$ solves (3.2).

Since $\alpha_{k}=o\left(\beta_{k}^{2}\right)$, we may assume that $\alpha_{k} \leq \beta_{k}^{2}$ for all $k \geq k_{0}$. From (3.6), for all $k \geq k_{0}$, we have

$$
\gamma\left(1-\gamma\|A\|^{2}\right)\left\|A x_{k}-S z_{k}\right\|^{2} \leq\left\|x_{k}-x_{k+1}\right\| M_{2}+\alpha_{k}\left\|f\left(y_{k}\right)\right\|^{2}+2 \alpha_{k}\left\|f\left(y_{k}\right)\right\|\left\|y_{k}-z\right\|,
$$

where $M_{2}:=\sup \left\{\left\|x_{k}-z\right\|+\left\|x_{k+1}-z\right\|: k \geq k_{0}\right\}$. This implies that, for $k \geq k_{0}$,

$$
\gamma\left(2-\gamma\|A\|^{2}\right) \frac{\left\|A x_{k}-S z_{k}\right\|^{2}}{\beta_{k}^{2}} \leq \frac{\left\|x_{k}-x_{k+1}\right\|}{\beta_{k}^{2}} M_{3}+\frac{\alpha_{k}}{\beta_{k}^{2}} M_{3} \leq \frac{\left\|x_{k}-x_{k+1}\right\|}{\alpha_{k}} M_{3}+\frac{\alpha_{k}}{\beta_{k}^{2}} M_{3},
$$

where $M_{3}:=\max \left\{M_{2}, \sup _{k \geq 1}\left\{\left\|f\left(y_{k}\right)\right\|^{2}+2\left\|f\left(y_{k}\right)\right\|\left\|y_{k}-z\right\|\right\}\right\}$. Subsequently, since $\| x_{k+1}-$ $x_{k} \|=o\left(\alpha_{k}\right), \alpha_{k}=o\left(\beta_{k}^{2}\right)$, and $\gamma\left(2-\gamma\|A\|^{2}\right)>0$, we have

$$
\lim _{k \rightarrow \infty} \frac{\left\|A x_{k}-S z_{k}\right\|}{\beta_{k}}=0
$$

For $k \geq k_{0}$, we compute

$$
\begin{aligned}
\left\|S z_{k}-S A z\right\|^{2} \leq & \left\|A x_{k}-\beta_{k} h\left(A x_{k}\right)-A z\right\|^{2} \\
\leq & \left\|A x_{k}-A z\right\|^{2}+2 \beta_{k}\left\langle h\left(A x_{k}\right), A z-z_{k}\right\rangle \\
= & \left\|A x_{k}-A z\right\|^{2}+2 \beta_{k}\left\langle h\left(A x_{k}\right), A z-A x_{k}\right\rangle+2 \beta_{k}^{2}\left\|h\left(A x_{k}\right)\right\|^{2} \\
= & \left\|A x_{k}-A z\right\|^{2}-2 \beta_{k}\left\langle A x_{k}-A z, h\left(A x_{k}\right)-h(A z)\right\rangle \\
& +2 \beta_{k}\left\langle h(A z), A z-A x_{k}\right\rangle+2 \beta_{k}^{2}\left\|h\left(A x_{k}\right)\right\|^{2} \\
\leq & \left\|A x_{k}-A z\right\|^{2}+\beta_{k}^{2}\left\|h\left(A x_{k}\right)-h(A z)\right\|^{2}+2 \beta_{k}\left\langle h(A z), A z-A x_{k}\right\rangle \\
& +2 \beta_{k}^{2}\left\|h\left(A x_{k}\right)\right\|^{2} .
\end{aligned}
$$


This gives

$$
\begin{aligned}
2\left\langle h(A z), A x_{k}-A z\right\rangle \leq & \frac{1}{\beta_{k}}\left(\left\|A x_{k}-A z\right\|^{2}-\left\|S z_{k}-S A z\right\|^{2}\right)+\beta_{k}\left\|h\left(A x_{k}\right)-h(A z)\right\|^{2} \\
& +2 \beta_{k}\left\|h\left(A x_{k}\right)\right\|^{2} \\
\leq & \frac{\left\|A x_{k}-S z_{k}\right\|}{\beta_{k}} M_{4}+\beta_{k}\left\|h\left(A x_{k}\right)-h(A z)\right\|^{2}+2 \beta_{k}\left\|h\left(A x_{k}\right)\right\|^{2},
\end{aligned}
$$

where $M_{4}:=\sup \left\{\left\|A x_{k}-A z\right\|+\left\|S z_{k}-S A z\right\|: k \geq 1\right\}<\infty$. Using this together with $A x_{k_{j}} \rightarrow$ $A q \in \operatorname{Fix}(S)$ and (3.14), we obtain $\langle h(A z), A q-A z\rangle \leq 0$ for all $A z \in \operatorname{Fix}(S)$. Thus, $A q \in \operatorname{Fix}(S)$ solves (3.2), and therefore, $q \in \Gamma$.

Finally, it remains to show that $x_{k} \rightarrow q \in \Gamma$. By the boundedness of $\left\{x_{k}\right\}_{k=1}^{\infty}$, it suffices to show that there is no subsequence $\left\{x_{k_{i}}\right\}_{i=1}^{\infty}$ of $\left\{x_{k}\right\}_{k=1}^{\infty}$ such that $x_{k_{i}} \rightarrow p \in H_{1}$ and $p \neq q$.

Indeed, if this is not true, the well-known Opial theorem would imply

$$
\begin{aligned}
\lim _{k \rightarrow \infty}\left\|x_{k}-p\right\| & =\lim _{j \rightarrow \infty}\left\|x_{k_{j}}-q\right\| \\
& <\lim _{j \rightarrow \infty}\left\|x_{k_{j}}-p\right\| \\
& =\lim _{k \rightarrow \infty}\left\|x_{k}-p\right\| \\
& =\lim _{i \rightarrow \infty}\left\|x_{k_{i}}-p\right\| \\
& <\lim _{i \rightarrow \infty}\left\|x_{k_{i}}-q\right\| \\
& =\lim _{k \rightarrow \infty}\left\|x_{k}-p\right\|,
\end{aligned}
$$

which leads to a contradiction. Therefore, the sequence $\left\{x_{k}\right\}_{k=1}^{\infty}$ converges weakly to a point $q \in \Gamma$.

\subsection{Split hierarchical variational inequality problems}

We consider another kind of split hierarchical variational inequality problem (in short, SHVIP) in which we consider a variational inequality formulation instead of the Minty variational inequality. More precisely, we consider the following split hierarchical variational inequality problem: find a point $x^{*} \in \operatorname{Fix}(T)$ such that

$$
\left\langle f\left(x^{*}\right), x-x^{*}\right\rangle \geq 0, \quad \text { for all } x \in \operatorname{Fix}(T),
$$

such that $A x^{*} \in \operatorname{Fix}(S)$ and it satisfies

$$
\left\langle h\left(A x^{*}\right), y-A x^{*}\right\rangle \geq 0, \quad \text { for all } y \in \operatorname{Fix}(S) .
$$

The solution set of the SHVIP is denoted by $\Omega$. Since $\operatorname{Fix}(T)$ and $\operatorname{Fix}(S)$ are nonempty closed convex and $f$ and $h$ are monotone and continuous, by the Minty lemma [13, Lemma 1], SHVIP (3.16)-(3.17) and SHMVIP (3.1)-(3.2) are equivalent. Hence, Algorithm 3.1 and Theorem 3.3 are also applicable for SHVIP (3.16)-(3.17).

When $\operatorname{Fix}(T)=C$ a closed convex subset of a Hilbert space $H_{1}$ and $\operatorname{Fix}(S)=Q$ a closed convex subset of a Hilbert space $H_{2}$, then SHVIP (3.16)-(3.17) is considered and studied by Censor et al. [24]. 
Remark 3.4 It is worth to note that, in the context of SHVIP (3.16)-(3.17), if we assume either the finite dimensional settings of $H_{1}$ and $H_{2}$ or the compactness of spaces $H_{1}$ and $H_{2}$, then the monotonicity assumptions of operators $f$ and $h$ can be omitted.

In fact, as in the statement and the proof of Theorem 3.3, since we know that $x_{k_{j}} \rightarrow q \in$ $\operatorname{Fix}(T)$, we also have $x_{k_{j}} \rightarrow q \in \operatorname{Fix}(T)$. Recalling the inequality (3.13), we have, for $k \geq k_{0}$,

$$
\left\|x_{k+1}-z\right\|^{2} \leq\left\|x_{k}-z\right\|^{2}+2 \alpha_{k}\left\langle f\left(y_{k}\right), z-y_{k}\right\rangle+2 \alpha_{k}^{2}\left\|f\left(y_{k}\right)\right\|^{2}
$$

which is equivalent to

$$
2\left\langle f\left(y_{k}\right), y_{k}-z\right\rangle \leq \frac{\left\|x_{k}-x_{k+1}\right\|}{\alpha_{k}} M+2 \alpha_{k}\left\|f\left(y_{k}\right)\right\|^{2},
$$

where $M:=\sup \left\{\left\|x_{k}-z\right\|+\left\|x_{k+1}-z\right\|: k \geq 1\right\}<\infty$. Since we know that $y_{k_{j}} \rightarrow q \in \operatorname{Fix}(T)$ and $f$ is continuous, by approaching $j$ to infinity, we obtain $\langle f(q), q-z\rangle \leq 0$, for all $z \in \operatorname{Fix}(T)$, which means that $q \in \operatorname{Fix}(T)$ solves (3.16). Similarly, from the inequality (3.15), we note that, for $k \geq k_{0}$,

$$
\left\|S z_{k}-S A z\right\|^{2} \leq\left\|A x_{k}-A z\right\|^{2}+2 \beta_{k}\left\langle h\left(A x_{k}\right), A z-A x_{k}\right\rangle+2 \beta_{k}^{2}\left\|h\left(A x_{k}\right)\right\|^{2},
$$

which is equivalent to

$$
2\left\langle h\left(A x_{k}\right), A x_{k}-A z\right\rangle \leq \frac{\left\|A x_{k}-S z_{k}\right\|}{\beta_{k}} M_{4}+2 \beta_{k}\left\|h\left(A x_{k}\right)\right\|^{2}
$$

where $M_{4}:=\sup \left\{\left\|A x_{k}-A z\right\|+\left\|S z_{k}-S A z\right\|: k \geq 1\right\}<\infty$. Since $A x_{k_{j}} \rightarrow A q \in \operatorname{Fix}(S)$ and $h$ is continuous, we also get $\langle h(A q), A q-A z\rangle \leq 0$, for all $A z \in \operatorname{Fix}(S)$, which means that $A q \in \operatorname{Fix}(S)$ solves (3.17), and subsequently, $q \in \Omega$.

Remark 3.5 The strong nonexpansiveness of operators $T$ and $S$ in Theorem 3.3 can be applied to the cases when the operators $T$ and $S$ are not only firmly nonexpansive, but with relaxation of being firmly nonexpansive and averaged nonexpansive but also strictly nonexpansive, that is, $\|T x-T y\|<\|x-y\|$ or $x-y=T x-T y$ for all $x, y$; regarding the compactness of the spaces $H_{1}$ and $H_{2}$, for more details, see [21, Remark 2.3.3].

\section{Some related problems}

In this section, we present some split-type problems which are special cases of SHVIP (3.1) and (3.2) and can be solved by using Algorithm 3.1.

\subsection{A split convex minimization problem}

Let $\phi: H_{1} \rightarrow \mathbb{R}$ and $\varphi: H_{2} \rightarrow \mathbb{R}$ be convex continuously differentiable functions and $A$ : $H_{1} \rightarrow H_{2}$ be a bounded linear operator such that $A\left(H_{1}\right) \cap \operatorname{Fix}(S) \neq \varnothing$. Consider the following split convex minimization problem (in short, SCMP): find

$$
x^{*} \in \operatorname{Fix}(T) \quad \text { such that } x^{*}=\underset{x \in \operatorname{Fix}(T)}{\operatorname{argmin}} \phi(x),
$$

and such that

$$
A x^{*} \in \operatorname{Fix}(S) \quad \text { such that } A x^{*}=\underset{y \in \operatorname{Fix}(S)}{\operatorname{argmin}} \varphi(y) .
$$


We know that the SCMP (4.1)-(4.2) can be formulated as the following split hierarchical variational inequality problem: find a point

$$
x^{*} \in \operatorname{Fix}(T) \text { such that }\left\langle\nabla \phi\left(x^{*}\right), x-x^{*}\right\rangle \geq 0, \quad \text { for all } x \in \operatorname{Fix}(T),
$$

and such that the point

$$
A x^{*} \in \operatorname{Fix}(S) \quad \text { such that }\left\langle\nabla \varphi\left(A x^{*}\right), y-A x^{*}\right\rangle \geq 0, \quad \text { for all } y \in \operatorname{Fix}(S) \text {, }
$$

where $\nabla \phi$ and $\nabla \varphi$ denote the gradient of $\phi$ and $\varphi$, respectively. Since $\nabla \phi$ and $\nabla \varphi$ are monotone [12, Proposition 5.3] and continuous, we can apply Algorithm 3.1 to obtain the solution of SCMP (4.1)-(4.2), and Theorem 3.3 will provide the convergence of the sequence $\left\{x_{k}\right\}_{k=1}^{\infty}$ to a solution of SCMP (4.1)-(4.2). Furthermore, in the context of finitedimensional cases, we can remove the convexity of $\phi$ and $\varphi$.

\subsection{A split variational inequality problem over the solution set of monotone variational inclusion problem}

Let $H_{1}$ and $H_{2}$ be two real Hilbert spaces, $\phi: H_{1} \rightarrow H_{1}, \varphi: H_{2} \rightarrow H_{2}$ be $L_{1}$, (respectively, $L_{2}$ )-inverse strongly monotone operators, $B_{1}: H_{1} \rightarrow 2^{H_{1}}, B_{2}: H_{2} \rightarrow 2^{H_{2}}$ are set-valued maximal monotone operators. Let us consider the following monotone variational inclusion problem (in short, MVIP): find

$$
x^{*} \in H_{1} \quad \text { such that } \quad 0 \in \phi\left(x^{*}\right)+B_{1}\left(x^{*}\right) \text {. }
$$

We denote the solution set of MVIP by $\operatorname{SOL}\left(\phi, B_{1}\right)$.

For $\sigma>0, \lambda \in\left(0,2 L_{1}\right)$, we know that the operator $J_{\sigma}^{B_{1}}(I-\lambda \phi)$ is an averaged nonexpansive operator and

$$
\operatorname{Fix}\left(J_{\sigma}^{B_{1}}(I-\lambda \phi)\right)=\operatorname{SOL}\left(\phi, B_{1}\right)
$$

where $J_{\sigma}^{B_{1}}$ represents the resolvent of $B_{1}$ with parameter $\sigma$.

We consider the following split variational inequality problem: find $x^{*} \in \operatorname{SOL}\left(\phi, B_{1}\right)$ such that

$$
\left\langle f(x), x-x^{*}\right\rangle \geq 0, \quad \text { for all } x \in \operatorname{SOL}\left(\phi, B_{1}\right)
$$

and such that $A x^{*} \in \operatorname{SOL}\left(\varphi, B_{2}\right)$ satisfies

$$
\left\langle h(y), y-A x^{*}\right\rangle \geq 0, \quad \text { for all } y \in \operatorname{SOL}\left(\varphi, B_{2}\right) .
$$

By using these facts and adding the assumption that $J_{\sigma}^{B_{2}}(I-\lambda \varphi)$ is cutter, we can apply Algorithm 3.1 and Theorem 3.3 to obtain the solution of SVIP (4.3)-(4.4).

\subsection{A split variational inequality problem over the solution set of equilibrium problem}

Let $H_{1}$ and $H_{2}$ be real Hilbert spaces, $C \subseteq H_{1}$ and $Q \subset H_{2}$ be nonempty closed convex sets, $\phi: C \times C \rightarrow \mathbb{R}$ and $\varphi: Q \times Q \rightarrow \mathbb{R}$ be bifunctions. The equilibrium problem defined by $C$ 
and $\phi$ is the problem of finding $x^{*} \in C$ such that

$$
\phi\left(x^{*}, x\right) \geq 0, \quad \text { for all } x \in C,
$$

and we denote the solution set of equilibrium problem of $C$ and $\phi$ by $\operatorname{EP}(C, \phi)$.

Recall that Blum and Oettli [26] showed that the bifunction $\phi$ satisfies the following conditions:

(A1) $\phi(x, x)=0$ for all $x \in C$;

(A2) $\phi$ is monotone, that is, $\phi(x, y)+\phi(y, x) \leq 0$ for all $x, y \in C$;

(A3) for all $x, y, z \in C$,

$$
\limsup _{t \downarrow 0} \phi(t z+(1-t) x, y) \leq \phi(x, y)
$$

(A4) for all $x \in C, \phi(x, \cdot)$ is a convex and lower semicontinuous function, and let $r>0$ and $z \in H_{1}$, then the set

$$
T_{r}(z):=\left\{x^{*} \in C: \phi\left(x^{*}, x\right)+\frac{1}{r}\left\langle x-x^{*}, x^{*}-x\right\rangle \geq 0 \text { for all } x \in C\right\} \neq \emptyset .
$$

Moreover, from [27], we know that

(i) $T_{r}$ is single-valued;

(ii) $T_{r}$ is firmly nonexpansive;

(iii) $\operatorname{Fix}\left(T_{r}\right)=\mathrm{EP}(C ; \phi)$.

We now consider the following split variational inequality problem: find $x^{*} \in \operatorname{EP}(C, \phi)$ such that

$$
\left\langle f(x), x-x^{*}\right\rangle \geq 0, \quad \text { for all } x \in \operatorname{EP}(C, \phi),
$$

and such that $A x^{*} \in \operatorname{EP}(Q, \varphi)$ satisfies

$$
\left\langle h(y), y-A x^{*}\right\rangle \geq 0, \quad \text { for all } y \in \operatorname{EP}(Q, \varphi) .
$$

Similarly, by assuming that conditions (A1)-(A4) in the context of the equilibrium problem defined by $Q$ and $\varphi$ hold, we see that, for all $u \in H_{2}$ and $s>0$, the set

$$
T_{s}(u):=\left\{y^{*} \in Q: \varphi\left(y^{*}, y\right)+\frac{1}{s}\left\langle y-y^{*}, y^{*}-u\right\rangle \geq 0 \text { for all } y \in Q\right\}
$$

is a nonempty set. Moreover, we also see that $T_{s}$ is single-valued and firmly nonexpansive; and $\operatorname{Fix}\left(T_{s}\right)=\operatorname{EP}(Q ; \varphi)$.

By using these facts, we obtain the result that the problem (4.5)-(4.6) forms a special case of the SHVIP (3.1)-(3.2). 


\section{Author details}

'Department of Mathematics, Aligarh Muslim University, Aligarh, 202 002, India. ${ }^{2}$ Department of Mathematics and Statistics, King Fahd University of Petroleum \& Minerals, Dhahran, Saudi Arabia. ${ }^{3}$ Department of Mathematics, Faculty of Science, Naresuan University, Phitsanulok, 65000, Thailand. ${ }^{4}$ Centre of Excellence in Mathematics, CHE, Si Ayutthaya Rd., Bangkok, 10400, Thailand.

\section{Acknowledgements}

The authors would like to thank the anonymous referees for their careful reading and suggestions, which allowed us to improve the first version of this paper. This research is partially support by the Center of Excellence in Mathematics the Commission on Higher Education, Thailand. QH Ansari was partially supported by a KFUPM Funded Research Project No. IN121037. N Nimana was supported by the Thailand Research Fund through the Royal Golden Jubilee PhD Program (Grant No. PHD/0079/2554)

Received: 14 May 2014 Accepted: 16 September 2014 Published: 13 October 2014

\section{References}

1. Censor, Y, Elfving, T: A multiprojection algorithm using Bregman projections in product space. Numer. Algorithms 8 , 221-239 (1994)

2. Byrne, C: Iterative oblique projection onto convex sets and the split feasibility problem. Inverse Probl. 18, 441-453 (2002)

3. Censor, Y, Elfving, T, Kopf, N, Bortfeld, T: The multiple-sets split feasibility problem and its applications for inverse problems. Inverse Probl. 21, 2071-2084 (2005)

4. Censor, Y, Bortfeld, T, Martin, B, Trofimov, A: A unified approach for inversion problems in intensity modulated radiation therapy. Phys. Med. Biol. 51, 2353-2365 (2006)

5. Xu, HK: Iterative methods for the split feasibility problem in infinite-dimensional Hilbert spaces. Inverse Probl. 26, $105018(2010)$

6. Xu, HK: Fixed point algorithms in convex optimization. In: Ansari, QH (ed.) Topics in Nonlinear Analysis and Optimization, pp. 87-136. World Publication, Delhi (2012)

7. Ansari, QH, Rehan, A: Split feasibility and fixed point problems. In: Ansari, QH (ed.) Nonlinear Analysis: Approximation Theory, Optimization and Applications. Springer, Berlin (2014)

8. Censor, Y, Segal, A: The split common fixed point problems for directed operators. J. Convex Anal. 16, 587-600 (2009)

9. Moudafi, A: The split common fixed-point problem for demicontractive mappings. Inverse Probl. 26, 1-6 (2010)

10. Ansari, QH, Lalitha, CS, Mehta, M: Generalized Convexity, Nonsmooth Variational Inequalities, and Nonsmooth Optimization. CRC Press, Boca Raton (2014)

11. Facchinei, F, Pang, J-S: Finite-Dimensional Variational Inequalities and Complementarity Problems, vols. I and II. Springer, New York (2003)

12. Kinderlehrer, D, Stampacchia, G: An Introduction to Variational Inequalities and Their Applications. Academic Press, New York (1980)

13. Minty, GJ: On the generalization of a direct method of the calculus of variations. Bull. Am. Math. Soc. 73, 314-321 (1967)

14. Ansari, QH, Ceng, L-C, Gupta, H: Triple hierarchical variational inequalities. In: Ansari, QH (ed.) Nonlinear Analysis: Approximation Theory, Optimization and Applications. Springer, Berlin (2014)

15. lemoto, S, Hishinuma, $\mathrm{K}$, liduka, $\mathrm{H}$ : Approximate solutions to variational inequality over the fixed point set of a strongly nonexpansive mapping. Fixed Point Theory Appl. 2014, Article ID 51 (2014)

16. liduka, $\mathrm{H}$ : A new iterative algorithm for the variational inequality problem over the fixed point set of a firmly nonexpansive mapping. Optimization 59, 873-885 (2010)

17. liduka, $\mathrm{H}$ : Fixed point optimization algorithm and its application to power control in CDMA data networks. Math. Program. 133, 227-242 (2012)

18. Jitpeera, T, Kumam, P: Algorithms for solving the variational inequality problem over the triple hierarchical problem. Abstr. Appl. Anal. 2012, Article ID 827156 (2012)

19. Yamada, I: The hybrid steepest descent method for the variational inequality problem over the intersection of fixed point sets of nonexpansive mappings. In: Butnariu, D, Censor, Y, Reich, S (eds.) Inherently Parallel Algorithms in Feasibility and Optimization and Their Applications, pp. 473-504. Elsevier, Amsterdam (2001)

20. Bruck, RE, Reich, S: Nonexpansive projections and resolvents of accretive operators in Banach spaces. Houst. J. Math. 3, 459-470 (1977)

21. Cegielski, A: Iterative Methods for Fixed Point Problems in Hilbert Spaces. Springer, New York (2012)

22. Opial, Z: Weak convergence of the sequence of successive approximations for nonexpansive mappings. Bull. Am. Math. Soc. 73, 591-597 (1976)

23. Polyak, BT: Introduction to Optimization. Optimization Software Inc., New York (1987)

24. Censor, Y, Gibali, A, Reich, S: Algorithms for the split variational inequality problem. Numer. Algorithms 59, 301-323 (2012)

25. Moudafi, A: Split monotone variational inclusions. J. Optim. Theory Appl. 150, 275-283 (2011)

26. Blum, E, Oettli, W: From optimization and variational inequalities. Math. Stud. 63, 123-146 (1994)

27. Combettes, PL, Hirstoaga, SA: Equilibrium programming in Hilbert spaces. J. Nonlinear Convex Anal. 6, 117-136 (2005)

doi:10.1186/1687-1812-2014-208

Cite this article as: Ansari et al.: Split hierarchical variational inequality problems and related problems. Fixed Point Theory and Applications 2014 2014:208. 